\title{
Paraneoplastic chorea: Case study with autopsy confirmation
}

\author{
Ivo W. Tremont-Lukats, Gregory N. Fuller, Teresa Ribalta, Pierre Giglio, and \\ Morris D. Groves ${ }^{1}$
}

\author{
Neuro-Oncology Department (I.W.T.-L., P.G., M.D.G.), Sections of Neuropathology and Immunocytochemistry \\ (G.N.F.), the University of Texas M.D. Anderson Cancer Center, Houston, TX 77030; and Anatomia \\ Patológica, Universitat de Barcelona, Barcelona, Spain (T.R.)
}

A 67-year-old man presented with a 7-month history of insidiously progressive chorea, ataxia, and vertigo. Neurologic examination revealed deficits referable to the basal nuclei, cerebellar vermis, and vestibular nuclei. Small-cell lung cancer was diagnosed by fine-needle biopsy of a parahilar mass. After chemotherapy, the patient's chorea worsened. Anti-Hu antibodies were present in serum and cerebrospinal fluid. Microscopic examination of the brain at autopsy revealed diffuse perivascular lymphocytic infiltrates, microglial activation, and neuronophagia throughout the neuraxis, including the brainstem, cerebellum, lenticular nuclei, striatum, and cerebral cortex. Prominent loss of Purkinje cells was seen in the cerebellar vermis and hemispheres to a lesser degree. Chorea is extremely rare as a paraneoplastic manifestation of cancer. The florid presentation and the positive findings contrasted with an unremarkable MRI of the brain. This case illustrates the preeminence of symptoms and signs over negative MRI findings in paraneoplastic encephalitis. Neuro-Oncology 4, 192-195, 2002 (Posted to NeuroOncology [serial online], Doc. 01-064, April 30, 2002. URL <neuro-oncology.mc.duke.edu>)

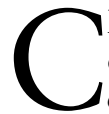
horea is a very rare symptom of the remote effects of cancer on the CNS. Few studies have described chorea as a presenting feature of paraneoplastic disease. In all but one of these studies, chorea has been

Received 4 December 2001, accepted 12 March 2002.

${ }^{1}$ Address correspondence and reprint requests to Morris D. Groves, Department of Neuro-Oncology, the University of Texas M.D. Anderson Cancer Center, Houston, TX 77030.

${ }^{2}$ Abbreviations used are as follows: $\mathrm{PE}$, paraneoplastic encephalitis; SCLC, small-cell lung carcinoma. correlated with MRI abnormalities. We report on a patient with SCLC $^{2}$ who had chorea as the dominant presenting symptom and whose MRI was normal. He had anti-Hu immunoreactivity in serum and cerebrospinal fluid with the characteristic histopathologic features of PE.

\section{Case Study}

\section{Clinical Course}

A 67-year-old man was evaluated in the Emergency Center of the M.D. Anderson Cancer Center for abnormal movements. These movements accidentally caused a pneumothorax during a needle biopsy of a right hilar mass. The patient reported a 7-month history of abnormal movements and vertigo accompanied by a weight loss of $22.5 \mathrm{~kg}$ over 4 months. The movements were involuntary, sudden, rapid but not ballistic, and consisted mainly of repeated shoulder shrug, as well as adduction and abduction movements of both legs. Initially, the movements were sporadic and self-limited, but became nearly continuous over the next several months. The movements ceased during sleep. Concomitantly, there was vertigo and gait unsteadiness. The patient described his experience of vertigo as a constant clockwise spinning sensation without nausea, vomiting, or visual changes. There was no exacerbation with head movements. The patient perceived his unsteadiness as a consequence of vertigo and avoided falling by widening his stance.

On exam, vital signs and oxygen saturation were normal. The mini-mental state exam score was $27 / 30$. There was nystagmus on horizontal and upward gaze. No other abnormalities were present upon examination of the cranial nerves. Myotatic reflexes were present but hypoactive throughout. There was a right plantar extensor 
response. Muscle tone was normal. He had abnormal, involuntary, almost continuous rapid movements of the neck, shoulders, and legs without ballismus or athetosis. The patient had a normal, age-adjusted perception to all sensory modalities including pain, temperature, soft touch, position sense, vibration, 2-point discrimination, stereognosis, and graphesthesia. Heel-knee and fingernose tests were normal. There was no disdiadochokinesis. The Romberg test was negative, but the patient had difficulty in keeping balance because of truncal ataxia. Stance was wide-based and gait was ataxic. Complete blood count, differential count, and serum chemistries were all normal. Anti-Yo and anti-Ri antibodies were negative. Anti-Hu antibodies were present in serum screening assays, confirmed with immunofluorescence and Western blot tests (Quest Diagnostics, San Juan Capistrano, Calif.). Biopsy of a lymph node in the right pulmonary hilum revealed poorly differentiated SCLC. A brain MRI (General Electric, Fairfield, Conn.; 1.5 Tesla) with contrast, diffusion-weighted images, and fluid-attenuated inversion recovery sequences showed no pertinent abnormalities. Clonazepam was started at $0.5 \mathrm{mg}$ by mouth 3 times daily for symptomatic control of movements.

Four days after admission, the patient received a first course of chemotherapy with cisplatin and etoposide and was discharged. Seven days later, he was readmitted because the choreic movements were worse. Clonazepam was changed to trihexyphenidyl and later to benztropine. Ten days after discharge, the patient was readmitted with uncontrollable chorea and agitation. The patient's family reported thrashing movements, paranoid ideation, and visual hallucinations. This mental status fluctuated prior to readmission. Haloperidol and benztropine exacerbated the choreic movements, and the patient appeared more restless and confused. Lorazepam i.v. mitigated the movements and the agitation. Lumbar puncture revealed an opening pressure of $130 \mathrm{~mm} \mathrm{H}_{2} \mathrm{O}$, a protein of $112 \mathrm{mg} / \mathrm{dl}$, normal cell count and differential, no oligoclonal bands, negative cultures, and an elevated cerebrospinal fluid anti-Hu antibody titer $(1: 1024)$. The patient developed neutropenia with an absolute neutrophil count $<500 / \mathrm{ml}$, sepsis, and multiple system failure, dying less than a month after the diagnosis of SCLC.

\section{Postmortem Examination}

At autopsy, SCLC metastases were identified in the right hilar, pretracheal, and mediastinal lymph nodes (Fig. 1). Serial sectioning and thorough macroscopic and microscopic examination of the lungs and other systemic organs failed to identify a primary site for the SCLC (occult primary site). Additional autopsy findings included bilateral aspiration pneumonia, pulmonary emphysema, hemorrhagic colitis of the ascending colon, severe atherosclerosis of the infrarenal abdominal aorta, and fibrinous pericarditis and pleuritis.

Brain examination at autopsy revealed the characteristic histopathologic features associated with PE: prominent perivascular lymphocytic infiltrates, astrogliosis, diffuse microglial activation with scattered microglial nodules associated with neuronophagia, and neuronal dropout (Fig. 2A-2C). Neuronal loss was particularly

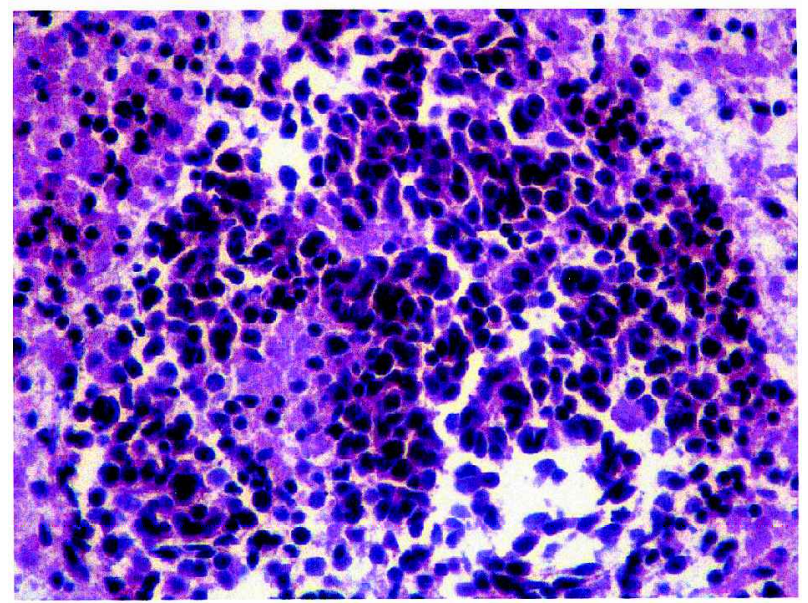

Fig. 1. Squash preparation of parahilar lymph node from patient with small cell carcinoma. Stained with hematoxylin and eosin. Original magnification $\times 100$.

striking in the striatum and in the cerebellar vermis, with marked loss of Purkinje cells (Fig. 2D). Loss of Purkinje cells in the cerebellar hemispheres was less severe than in the vermis. Examination of the right temporal lobe showed a white matter cyst and mild astrogliosis. There was no evidence of metastatic SCLC to the CNS.

\section{Discussion}

This case highlights the recognition of chorea as a paraneoplastic movement disorder in SCLC. Posner (1995) estimated that less than $1 \%$ of patients with cancer have a clinically significant paraneoplastic syndrome. In 3843 patients with lung cancer, only $0.36 \%$ had paraneoplastic encephalomyelitis, based on clinical examination (Henson and Urich, 1982). A literature search of MEDLINE, CANCERLIT, and Current Contents databases from 1966 through February 22, 2002, using the terms "paraneoplastic syndromes/diseases," "small cell lung cancer," and "chorea" as keywords, identified 5 case reports, one of which reported SCLC with anti-Hu antibodies (Heckmann et al., 1997). The first known published report of paraneoplastic chorea involved a patient with SCLC who also had dystonia, sensory dysfunction, nystagmus, and ataxia. This patient had a normal MRI, and autoantibodies were not determined at that time (Albin et al., 1988) (Table 1). Compared with other cases, our patient did not have solid clinical evidence of cognitive dysfunction. The cause of delirium prompting his readmission will never be known with certainty, but could have been due to an accelerated worsening of his paraneoplastic condition, the use of anticholinergic and antipsychotic drugs in an aging patient, or intercurrent sepsis. Chorea has also rarely been reported as part of a paraneoplastic syndrome associated with other solid tumors and hematologic malignancies, with antineuronal antibodies different from anti-Hu (Batchelor et al., 1998; Kujawa et al., 2001; Tani et al., 2000).

Neuro-Oncology a JULY 2002 


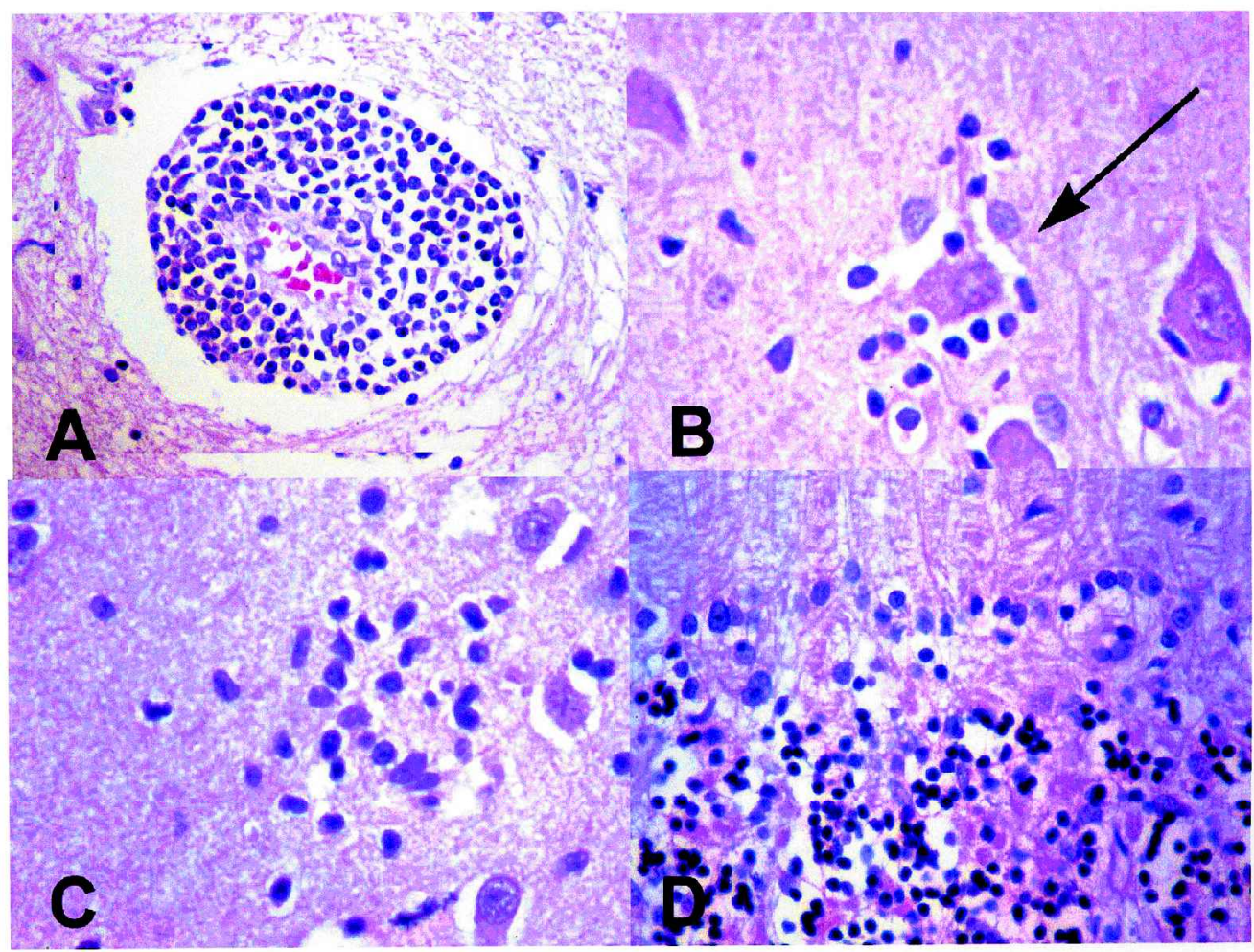

Fig. 2. Hematoxylin and eosin-stained sections of striatum and cerebellum of a patient with chorea and paraneoplastic encephalitis. A. Dense perivascular lymphocytic infiltrate. B. Perineuronal lymphocytic satellitosis. C. Microglial nodule. D. High-powered microscopic examination of the cerebellar cortex (vermis) at the junction of the molecular and granular cell layers showing loss of Purkinje cells. Original magnification $A-D, \times 400$.

Table 1. All known patients with limited-stage SCLC and paraneoplastic chorea

\begin{tabular}{|c|c|c|c|c|c|}
\hline Literature & $\begin{array}{c}\text { Onset } \\
\text { before } \\
\text { diagnosis } \\
\text { of primary } \\
\text { cancer }\end{array}$ & Findings & CSF abnormalities & Autoantibody & MRI \\
\hline Albin et al. (1988) & Yes & $\begin{array}{l}\text { Chorea, encephalopathy, ataxia, } \\
\text { nystagmus, sensory neuropathy, } \\
\text { cranial nerve neuropathy }\end{array}$ & $\begin{array}{l}\text { Protein } 20-60 \mathrm{mg} / \mathrm{dl} \\
\text { OCB+ }\end{array}$ & Not determined & Normal \\
\hline Heckmann et al. (1997) & Yes & $\begin{array}{l}\text { Chorea, encephalopathy, cerebellar } \\
\text { dysfunction, sensory neuropathy }\end{array}$ & $\begin{array}{l}\text { Protein } 55 \mathrm{mg} / \mathrm{dl} \text {, } \\
\text { WBC: } 25 / \mathrm{ml}\end{array}$ & Anti-Hu+ & Caudate atrophy \\
\hline Croteau et al. (2001) & Yes & $\begin{array}{l}\text { Chorea, encephalopathy, } \\
\text { cerebellar dysfunction, vitreitis } \\
\text { and papillitis (?) }\end{array}$ & $\begin{array}{l}\text { Protein } 83 \mathrm{mg} / \mathrm{dl} \text {, } \\
\text { WBC: } 15 / \mathrm{ml} \\
\text { OCB+ }\end{array}$ & $\begin{array}{l}\text { Anti } 76-k D \\
\text { neuronal antigen } \\
\text { Anti } \mathrm{Hu}-\end{array}$ & $\begin{array}{l}\text { Hyperintense } \\
\text { signals in } \\
\text { neostriatum }\end{array}$ \\
\hline Present report & Yes & Chorea, nystagmus, ataxia & $\begin{array}{l}\text { Protein } 105 \mathrm{mg} / \mathrm{dl} \text {, } \\
\text { WBC: } 6 / \mathrm{ml} \\
\text { OCB- }\end{array}$ & Anti-Hu+ & $\begin{array}{l}\text { No pertinent } \\
\text { abnormalities }\end{array}$ \\
\hline
\end{tabular}

Abbreviations: CSF, cerebrospinal fluid; $O C B$, oligoclonal bands; WBC, white blood cells.

Unlike the cases reported by Albin et al. (1988) and Heckmann et al. (1997), our patient never had clinical findings indicative of sensory dysfunction at any level of the nervous system. In that aspect, the patient reported by Croteau et al. (2001) (Anti-Hu negative) is more similar to our case.
The clinical and radiologic presentations of PE vary. MR scans can be normal earlier, but with more advanced or severe symptoms, diffuse cerebellar atrophy and hyperintense signals on T2-weighted images can appear in the white matter and basal nuclei. Despite 7 months of progressive and disabling symptoms, a brain MR scan 
obtained under general anesthesia did not yield pertinent findings.

The characteristic neuropathologic findings of $\mathrm{PE}$ include a variable degree of neuronal loss, diffuse microglial activation, multifocal microglial nodules, neuronophagia, astrocytosis, and perivascular lymphocytic cuffing (Dalmau et al., 1999; Graus et al., 1990, 2001; Scaravilli et al., 1999). These changes may involve virtually the entire neuraxis to a variable degree, constituting an encephalomyeloganglioradiculitis, although specific regions may be affected solely or predominantly in isolation, with clinical symptoms reflecting the area(s) involved. The regions most frequently involved by PE are the cerebral gray matter (hippocampal formation, hypothalamus, basal ganglia, and neocortex), brainstem (particularly the gray matter along the floor of the 4th ventricle and the inferior olivary nuclei of the medulla), dentate nuclei of the cerebellum, spinal cord (anterior horns), posterior nerve roots, and dorsal root ganglia.
Foci of perivascular lymphocytic infiltration are also often observed in the leptomeninges. $\mathrm{CD} 8^{+} \mathrm{T}$ cells predominate in the inflammatory cell infiltrates of PE, particularly in those infiltrates that surround degenerating neurons. An extensive loss of cerebellar Purkinje cells without accompanying inflammatory cell infiltrates and a less conspicuous loss of granule cells is observed in cases of paraneoplastic cerebellar degeneration and also occurs as part of the spectrum of $\mathrm{PE}$ as seen in this case.

The overall prognosis of paraneoplastic chorea is bad, with occasional exceptions. Croteau et al. (2001) have described a patient with chorea related to SCLC whose clinical and immunologic response paralleled that of his primary tumor in which almost complete disappearance of the brain lesions were seen on MRI. No other case has had such a striking correlation between tumor response and immunologic response with clinical improvement. Our patient had a totally different outcome, with death attributable to hematologic toxicity and sepsis.

\section{References}

Albin, R.L., Bromberg, M.B., Penney, J.B., and Knapp. R. (1988) Chorea and dystonia: A remote effect of carcinoma. Mov. Disord. 3, 162-169.

Batchelor, T.T., Platten, M., Palmer-Toy, D.E., Hunter, G.J., Lev, M.H., Dalman, J., and Hochberg, F.H. (1998) Chorea as a paraneoplastic complication of Hodgkin's disease. J. Neurooncol. 36, 185-190.

Croteau, D., Owainati, A., Dalmau, J., and Rogers, L.R. (2001) Response to cancer therapy in a patient with a paraneoplastic choreiform disorder. Neurology 57, 719-722.

Dalmau, J., Gultekin, H.S., and Posner, J.B. (1999) Paraneoplastic neurologic syndromes: Pathogenesis and physiopathology. Brain Pathol. 9, 275-284.

Graus, F., Ribalta, T., Campo, E., Monforte, R., Urbano, A., and Rozman, C. (1990) Immunohistochemical analysis of the immune reaction in the nervous system in paraneoplastic encephalomyelitis. Neurology 40, 219-222.

Graus, F., Keime-Guibert, F., Rene, R., Benyahia, B., Ribalta, T., Ascaso, C., Escaramis, G., and Delattre, J.Y. (2001) Anti-Hu-associated paraneoplastic encephalomyelitis: Analysis of 200 patients. Brain 124, 1138-1148.
Heckmann, J.G., Lang, C.J., Druschky, A., Claus, D., Bartels, O., and Neundorfer, B. (1997) Chorea resulting from paraneoplastic encephalitis. Mov. Disord. 12, 464-466.

Henson, R.A., and Urich, H. (1982) Cancer and the Nervous System: The Neurological Manifestations of Systemic Malignant Disease.Oxford: Blackwell Scientific.

Kujawa, K.A., Niemi, V.R., Tomasi, M.A., Mayer, N.W., Cochran, E., and Goetz, C.G. (2001) Ballistic-choreic movements as the presenting feature of renal cancer. Arch. Neurol. 58, 1133-1135.

Posner, J.B. (1995) Neurologic Complications of Cancer. Contemporary Neurology Series 45. Philadelphia: F.A. Davis. pp. 353-385.

Scaravilli, F., An, S.F., Groves, M., and Thom, M. (1999) The neuropathology of paraneoplastic syndromes. Brain Pathol. 9, 251-260.

Tani, T., Piao, Y., Mori, S., Ishihara, N., Tanaka, K.I., Wakabayashi, K., and Takahashi, H. (2000) Chorea resulting from paraneoplastic striatal encephalitis. J. Neurol. Neurosurg. Psychiatry 69, 512-515. 